\title{
A CONDIÇÃO DAS COXAS ANTERIORES, DO MESO-CATEPIMERO E DO MESO-CATEPISTERNO DOS TABANIDAE (DIPTERA) E SUA POSSIVEL IMPORTÂNCIA NA CLASSIFICAÇÃO
}

\author{
Nelson Bernardi ${ }^{1}$
}

\begin{abstract}
The condition of certain neglected structures and their possible importance to the classification of the family Tabanidae are studied. The front coxae are considered as belonging to two types: 1) short and robust; 2) long and slender. The posterior edge of the meso-katepimeron presents two conditions: 1) without projection, 2) with backward projection. Similar conditions are found in the meso-Katepisternum, but with intermediate conditions. These characters are discussed in relation to the system proposed for the family by Mackerras. Conditions 1 and 2 of the former two structures separate, respectively, the Pangoniinae and Scepsidinae from the Chrysopsinae and Tabaninae. Condition 2 of the third structure seems to be developing independently in different groups of Tabaninae, with varying degrees of development.
\end{abstract}

\section{INTRODUÇÃO}

Com freqüência, os tabanidologistas têm lamentado a pobreza de caracteres estruturais da família. Embora esse problema não seja peculiar à família Tabanidae, como podem testemunhar especialistas em muitos outros grupos, trata-se de importante desafio a ser enfrentado nos níveis genérico e supra-genérico, especialmente na subfamilia Tabaninae. A meu ver, boas provas dessa dificuldade encontram-se no elevado número de subgeneros tradicionalmente adotados pelos tabanidologistas, muitos dos quais indubitavelmente artificiais, e no fato de os caracteres básicos utilizados para separar as tribos Diachlorini e Tabanini, da subfamília Tabaninae, não serem realmente diagnósticos, especialmente na Região Neotropical (Fairchild, 1969).

Mackerras (1955a), comentando os problemas envolvidos na delimitação dos gêneros de Tabanidae, afirma: "This is a situation witch, I believe, no amount of searching for new characters will overcome". A afirmação de Mackerras, provavelmente o pesquisador que mais seriamente atacou esses problemas, é sintomática, embora não inclua, creio eu, a implicação de que a busca contínua de nóvos caracteres seja desnecessária. As próprias pesquisas de Mackerras (1954, 1955a, 1955b), trouxeram à luz novos e importantes caracteres morfológicos.

O presente trabalho tem por finalidade apresentar algumas observações novas. Os caracteres aqui propostos não devem ser considerados definitivos, mas precisam ser testados. A avaliação de um novo caráter é sempre necessária, especialmente quando se pretende que esse caráter se aplique a grupos grandes e de distribuição mundial: Philip, em 1941, lançou mão de dois caracteres que vêm sendo amplamente usados desde então, da

1. Departamento de Zoologia, Instituto de Biocências, Universidade Estadual Paulista, 18610 Botucatu, São Paulo. 
seguinte forma: basicosta com setas tão abundantes quando na área costal adjacente (Tabanini) ou sem tais setas (Diachlorini). Esses caracteres, contudo, apresentam problemas, especialmente com relação às formas neotrópicas, o que obrigou Fairchild (1969) a empregar certas combinações de caracteres para a delimitação dos grupos em questão, mas com sucesso apenas relativo. Se já como diagnósticos esses caracteres não são consistentes, são ainda mais problemáticos para o esclarecimento das relações filogenéticas.

Ainda quanto à procura de novos caracteres, cumpre mencionar um importante trabalho de Stuckenberg (1973), onde o autor, embora que não primordialmente interessado na classificação interna dos Tabanidae, estudou caracteres que me parecem, após rápido exame de alguris quantos exemplares, poderem levar a bons resultados quando mais amplamente utilizados na família.

Finalmente, devo lembrar que não procuro caracteres que possam ser facilmente usados na identificação, mas que forneçam informações importantes para a classificação e o esclarecimento das relações filogenéticas. Não há novidade alguma nisso, uma vez que alguns dos principais caracteres genitais usados por Mackerras $(1954,1955 \mathrm{a}, 1955 \mathrm{~b})$ e outros autores não são facilmente utilizados nas práticas rotineiras de identificação. Se a identificação é facilitada por novos caracteres maiores, tanto melhor. Com alguma experiência, entretanto, creio que os caracteres apresentados a seguir se mostrarão úteis também para a identificação.

0 thaterial que estudei é quase todo da Região Neotropical. Examinei várias dezenas de etpécies de todas as tribos e quase todos os gêneros da Região reconhecidos por Fairchild (1969). De outras regiões, examinei exemplares dos seguintes gêneros: Agkistrocerus, Apatolestes, Atylotus, Chrysops, Goniops, Haematopota, Hamatabanus, Hybomitra, Pangonius, Pilimas, Silvius, Stonemyia, Tabanus e Whitneyomiyia.

A forma das coxas anteriores é suficiente para dividir a família em dois grandes grupos. Os Pangoniinae (Figs. 1-2) e Sc sidinae (Fig. 3) têm essas coxas curtas e robustas, enquanto os Chrysopsinae (Fig. 4) e Tabaninae (Figs. 5-6) as têm mais longas e del. gadas. Na prática, essas diferenças são facilmente aprendidas e com pouca experiência pode-se, observando as coxas anteriores, saber se um exemplar pertence a qualquer desses dois grupos. Mesmo em exemplares com farta pilosidade na região em questão, podese, com alguma experiência, determinar a condição das coxas. Embora a Figura 1 possa dar a impressão de um certo alongamen ', o exame dos exemplares não deixará dúvida. A condição encontrada nos Pelecorhynchidae e Pangoniinae é, a meu ver, a primitiva.

Para a análise das estruturas mesotorácicas, basta tomar como referência o trabalho de BARRETTO (1946), onde são encontradas outras referências úteis e onde o autor busca uniformizar, tanto quanto possível, a nomenclatura.

Resumidamente, temos a seguinte: a) a mesopleura é dividida pela sutura mesopleural em mesepisterno e mesepimero; b) o mesepisterno é dividido pela sutura episternal em meso-anepisterno e meso-catepisterno; c) o mesepimero é dividido pela śutura epimeral em meso-anepimero e meso-catepimero; d) o meso-catepimero é fundido com o mero da coxa média, compondo o chamado meropleurito. 0 quadro descrito pode ser visto na figura 7 .

Interessa-nos aqui a forma assumida pelo bordo posterior do meso-catepisterno e do meso-catepimero.

Quanto ao meso-catepimero, nota-se que há ou não uma projeção posterior. Essa diferença mostrou-se consistente no material estudado, podendo ser assim resumida:

meso-catepimero sem projeção: Pangoniidae, Scepsidinae.

meso-catepimero com projeção: Chrysopsinae, Tabaninae. 


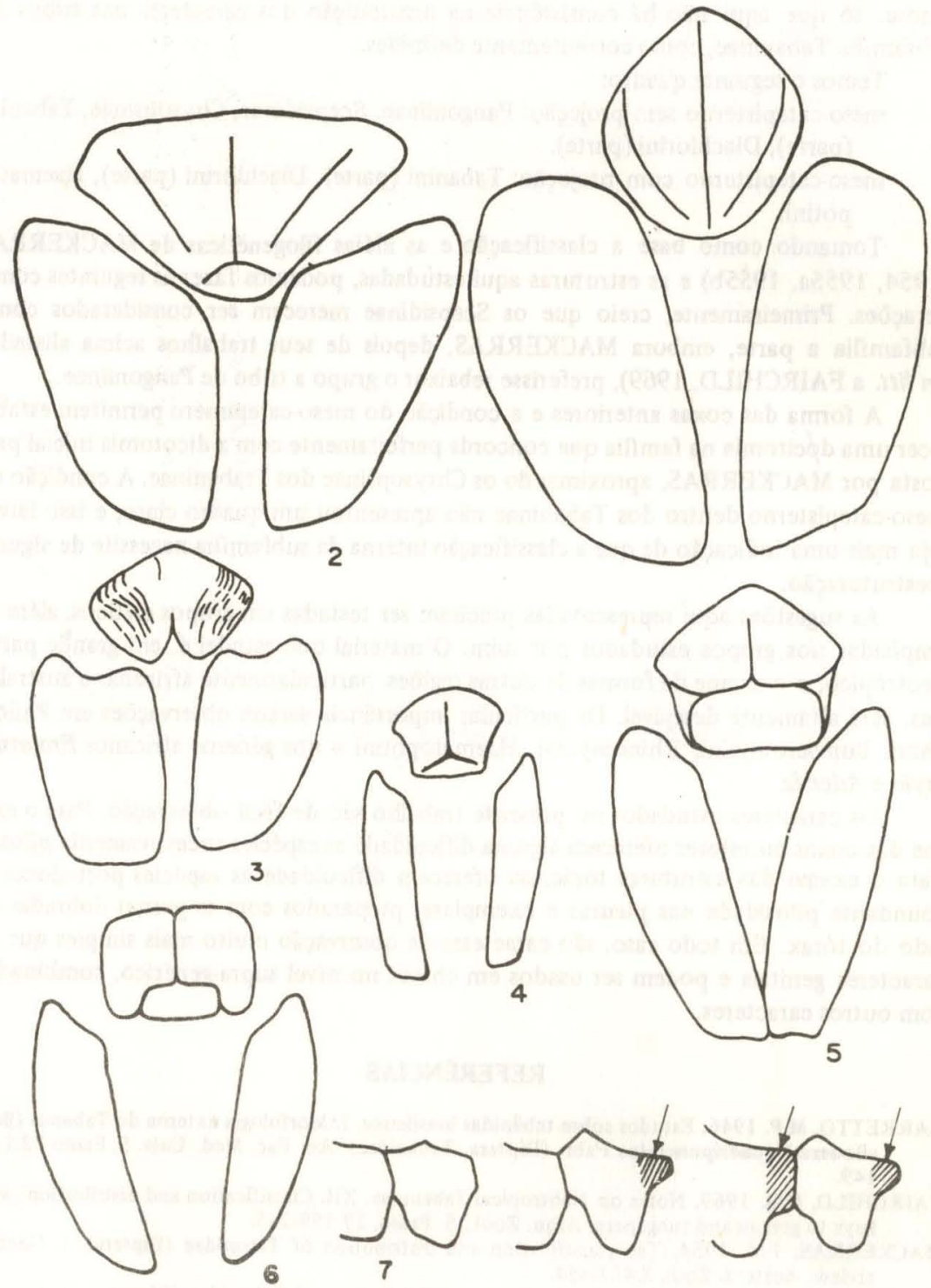

Figuras 1 a 7: Vista frontal das coxas anteriores. 1. Fidena morio. 2. Goniops chrysocoma. 3. Scepsis nivalis. 4. Chrysops brunneus. 5. Acanthocera anacantha. 6. Bolbodimyia brunneipenis. 7. Em cada desenho, a metade esquerda representa o catepisterno, a direita catepigero. Os desenhos são esquemáticos e as partes assinaladas indicam estruturas comentadas no texto. À esquerda, Pangoniinae; no meio, Chry sopsinae; à direita, Tabaninae $c 0 m$ as qualificap̧öes do texto. 
Examinando a forma do meso-catepisterno, observamos uma modificação semelhante, só que aqui não há consistência na distribuição dos caracteres nas tribos da subfamília Tabaninae, como correntemente definidas.

Temos o seguinte quadro:

meso-catepisterno sem projeção: Pangoniinae, Scepsidinae, Chysopsinae, Tabanini (parte), Diachlorini (parte).

meso-catepisterno com projeção: Tabanini (parte), Diachlorini (parte), Haematopotini.

Tomando como base a classificação e as idéias filogenéticas de MACKERRAS (1954, 1955a, 1955b) e as estruturas aqui estudadas, podemos fazer as seguintes considerações. Primeiramente, creio que os Scepsidinae merecem ser considerados como subfamília a parte, embora MACKERRAS, depois de seus trabalhos acima alistados (in litt. a FAIRCHILD, 1969), preferisse rebaixar o grupo a tribo de Pangoniinae.

A forma das coxas anteriores e a condição do meso-catepimero permitem estabelecer uma d $\oint$ citomia na familia que concorda perfeitamente com a dicotomia inicial proposta por MACKERRAS, aproximando os Chrysopsinae dos Trabaninae. A condição do meso-catepisterno dentro dos Tabaninae não apresentou um quadro claro, e isso talvez seja mais uma indicação de que a classificação interna da subfamília necessite de alguma reestruturação.

As sugestões aqui representadas precisam ser testadas em grupos críticos, além de ampliadas nos grupos estudados por mim. O material que estudei é, em granlle parte, neotrópico, e o exame de formas de outras regiões, particularmente africanas e australianas, será altamente desejável. De particular importância seriam observações em Philolichini, Bonvieromyiini, Rhinomyzini, Haematopotini e nos gêneros africanos Braunsiomyia e Adersia.

Os caracteres estudados no presente trabalho são de fácil observação. Para o exame das coxas anteriores oferecem alguma dificuldade as espécies excessivamente pilosas. Para o exame das estruturas torácicas oferecem dificuldade as espécies portadoras de abundante pilosidade nas pleuras e exemplares preparados com as pernas dobradas ao lado do tórax. Em todo caso, são caracteres de observação muito mais simples que os caracteres genitais e podem ser usados em chaves no nível supra-genérico, combinados com outros caracteres.

\section{REFERENCIAS}

BARRETTO, M.P. 1946. Estudos sobre tabânidas brasileiros. I. Morfologia externa do Tabanus (Boeciloderas) quadripunctatus Fabr. (Diptera, Tabanidae). An. Fac. Med. Univ. S. Paulo 22:113 149.

FAIRCHILD, G.B. 1969. Notes on Neotropical Tabanidae. XII. Classification and distribution, with keys to genera and subgenera. Arqu. Zool., S. Paulo, 17:199-255.

MACKERRAS, I.M. 1954. The classification and distribution of Tabanidae (Diptera). I. General review. Austr. J. Zool. 2:431-454.

MACKERRAS, I.M. 1955a. The classification and distribution of Tabanidae (Diptera). II. History: Morphology: Classification: Subfamily Pangoniinae. Austr. J. Zool. 3:439-511.

MACKERRAS, I.M. 1955b. The classification and distribution of Tabanidae (Diptera). III. Subfamilies Scepsidinae and Chrysopinae. Austr. J. Zool. 3:583-633.

PHILIP, C.B. 1941. Comments on the supra-specific categories of Nearctic Tabanidae (Diptera). Can. Ent. 73:2-14.

STUCKENBERG, B.R. 1973. The Athericidae, a new family in the lower Brachycera (Diptera). Ann. Natal Mus. 21:649-673, 47 figs. 